8 Chapman EJ, Knowles MA. Necdin: a multi-functional protein with potential tumor suppressor role? Mol Carcinog 2009; 48: 975-981.

9 Tsai TF, Armstrong D, Beaud AL. Necdin-deficient mice do not show lethality or the obesity and infertility of Prader-Willi syndrome. Nat Genet 1999; 22: 15-16.
10 Meuwissen R, Linn SC, Linnoila RI, et al. Induction of small cell lung cancer by somatic inactivation of both Trp53 and Rb1 in a conditional mouse model. Cancer Cell 2003; 4: 181-189.

\title{
Clinical features of central airway involvement in autoimmune pancreatitis
}

\section{To the Editors:}

Autoimmune pancreatitis (AIP) is characterised by a high serum immunoglobulin (Ig)G4 concentration and various extrapancreatic complications, including those of the lung [1]. Therefore, AIP is currently not viewed as a separate disease entity, but as the pancreatic involvement of a systemic IgG4related disease [2]. We previously reported a patient with AIP showing central airway stenosis and bilateral hilar lymphadenopathy (BHL) similar to sarcoidosis [3]. Following this case, we prospectively identified an additional five patients with AIP who revealed similar airway findings (and BHL) in our department from September 2007 to January 2009. The six patients (including the first patient) met the diagnostic criteria of AIP proposed by Japanese Pancreatic Society in 2006 [1]. In order to update the available clinical and therapeutic information on central airway involvement in AIP, we performed airway biopsies and other examinations in these six patients.

All six patients gave their written informed consent for the performance of bronchoscopy and blood sampling. The angiotensin-converting enzyme (ACE) and IgG4 concentrations were measured at Special Reference Laboratories Inc. (Tokyo, Japan), and interleukin (IL)-6 was measured at Mitsubishi Kagaku Bio-Clinical Laboratories Inc. (Tokyo, Japan). Chest computed tomography (CT) was performed with a multidetector row helical CT scanner (LightSpeed VCT; GE Medical Systems, Milwaukee, WI, USA) with both inspiratory and expiratory views [4]. All CT images were reviewed by two radiologists (S. Kawakami and Y. Fujinaga, Shinshu University School of Medicine, Matsumoto, Japan) [4]. Fibreoptic bronchoscopy (BF 1T-240 or 1T-260; Olympus corporation, Tokyo, Japan) was performed, and bronchoalveolar lavage (BAL), transbronchial lung biopsy (TBLB) and bronchial biopsy were achieved according to our routine protocol [5]. The TBLB was carried out in the right upper lobe (S2 and S3) and the bronchial biopsy was performed at the right second spur and the upper lobe bronchi. Immunohistochemical staining was performed by affinity-purified sheep polyclonal antibody against human IgG4 (The Binding Site Group Limited, Birmingham, UK). The numbers of both IgG4- and IgG-positive cells were counted in regions of the highest cellular density and averaged for three high-power fields (HPFs) by one pathologist (T. Uehara) [6]. Wilcoxon signed-rank test was used to compare the measurements before and after treatment.
Patient profiles, laboratory data, chest CT findings, pulmonary function tests (PFTs) and bronchoscopic data are shown in table 1. Four out of our six patients with airway involvement associated with AIP were middle-aged men, similar to the epidemiological features described in our previous study on patients with AIP [1]. Cough without dyspnoea or fever was present in all six patients. Although serological tests did not show particular abnormalities in terms of lactate dehydrogenase (LDH), C-reactive protein (CRP), ACE and IL-6, the serum levels of IgG and IgG4 were extremely high in all patients (table 1). BHL was observed in all six patients. We have previously reported hilar lymphadenopathy to be present in $\sim 78 \%$ of the patients with AIP, as detected by chest CT [4]. The most characteristic finding on chest $\mathrm{CT}$ imaging was thickening of the bronchial wall and bronchovascular bundles (fig. 1a). The PFTs (table 1) showed no restrictive ventilatory defects; however, airflow limitation was observed in several patients (forced expiratory volume in $1 \mathrm{~s}$ (FEV1)/forced vital capacity (FVC) $<70 \%$ in four and $70-80 \%$ in two patients; median $69.7 \%$, range $60.5-77.6 \%$ ) and the median FEV1 was $2.87 \mathrm{~L}$ (range 1.56$3.60 \mathrm{~L}$ ). The bronchoscopic examinations showed irregular stenoses of the bronchus and bronchi accompanied by mucosal oedema and engorged vessels (fig. $1 \mathrm{~b}$ and c) in all patients. In the BAL fluid (BALF), there were high lymphocyte counts with CD4 predominance in all patients, increased eosinophil counts in four patients and low plasma cell counts in all patients. In three out of four patients, the eosinophil count was only slightly elevated. The bronchial biopsy specimens of all patients revealed diffuse airway inflammatory infiltrates, consisting mainly of plasma cells and lymphocytes, together with fibrosis. Although there were scattered eosinophils in the bronchial tissues of all patients, typical findings of asthma (i.e. goblet cell hyperplasia and subepithelial fibrosis) [7] were not observed in any of the bronchial biopsy specimens. Because airway hypersensitivity was not even observed in case 4 , who showed the highest number of eosinophils in BALF, we thought that these findings were a characteristic feature of airway involvement in AIP other than typical asthma. Moreover, these pathological findings of the airway were similar to the other extrapancreatic lesions in AIP [1]. The immunohistochemical examinations showed massive infiltrations of many IgG4positive plasma cells in the bronchial biopsy specimens in all patients. The TBLB specimens of three out of the six patients demonstrated many IgG4-positive plasma cells in the peripheral bronchus and bronchiole; however, few IgG4-positive plasma 


\begin{tabular}{|c|c|c|c|c|c|c|}
\hline $\begin{array}{l}\text { Patient profiles, } \\
\text { bronchoscopic d }\end{array}$ & tomogr & (CT) fi & gs, pulr & ary func & tests ( & and \\
\hline Case & 1 & 2 & 3 & 4 & 5 & 6 \\
\hline Age yrs & 63 & 50 & 50 & 55 & 60 & 78 \\
\hline Sex & $\mathrm{F}$ & M & M & M & M & $\mathrm{F}$ \\
\hline Smoking pack-yrs & 0 & 19 & 14 & 26.2 & 0 & 0 \\
\hline Respiratory symptom & Cough & Cough & Cough & Cough & Cough & Cough \\
\hline \multicolumn{7}{|l|}{ Serological tests (normal range) } \\
\hline $\mathrm{LDH}$ IU $\cdot \mathrm{mL}^{-1}(114-220)$ & 161 & 168 & 150 & 215 & 149 & 214 \\
\hline $\mathrm{CRP} m g \cdot \mathrm{dL}^{-1}(<0.1)$ & 0.03 & 0.04 & 0.13 & 0.09 & 0.03 & 0.01 \\
\hline ACE U.L $L^{-1}(8.3-21.4)$ & 5.3 & 7.8 & 17.3 & 19.5 & 14.6 & 19.0 \\
\hline $\mathrm{IL}-6 \mathrm{pg} \cdot \mathrm{mL}^{-1}(<2.41)$ & 1.27 & 0.30 & 2.02 & 1.52 & 0.54 & 0.38 \\
\hline $\mathrm{BHL}$ & + & + & + & + & + & + \\
\hline Bronchial wall and BVB thickening & + & + & + & + & + & + \\
\hline Small nodular shadow & - & + & - & + & + & + \\
\hline Pleural irregularity & - & + & - & + & + & + \\
\hline Interlobular septal thickening & - & - & - & - & - & + \\
\hline Consolidation & - & - & - & - & - & - \\
\hline Emphysematous change & - & - & - & + & - & - \\
\hline \multicolumn{7}{|l|}{ PFTs } \\
\hline VC L & 3.11 & 5.10 & 4.96 & 3.73 & 4.27 & 2.01 \\
\hline VC $\%$ pred & 129.0 & 138.2 & 130.5 & 106.0 & 100.7 & 82.4 \\
\hline FEV 1 L & 2.17 & 3.60 & 3.52 & 3.08 & 2.67 & 1.56 \\
\hline Neutrophils \% & 0.4 & 0.4 & 0.9 & 0.4 & 0.8 & 1.8 \\
\hline Eosinophils \% & 0 & 5.2 & 4.1 & 44.2 & 0.2 & 6.7 \\
\hline $\mathrm{CD} 4 / \mathrm{CD} 8$ ratio & 4.48 & 2.40 & 1.53 & 1.71 & 1.27 & 1.70 \\
\hline \multicolumn{7}{|c|}{ Immunohistochemical positive cells in bronchi } \\
\hline IgG4-positive cells per HPF & 80.0 & 80.6 & 54.3 & 71.6 & 43.0 & 36.6 \\
\hline IgG-positive cells per HPF & 126.0 & 111 & 64.3 & 107.6 & 86.0 & 51.6 \\
\hline IgG4-positive/lgG-positive cells \% & 63.4 & 72.6 & 84.4 & 34.5 & 50.0 & 70.9 \\
\hline
\end{tabular}

LDH: lactate dehydrogenase; CRP: C-reactive protein; ACE: angiotensin-converting enzyme; IL: interleukin; Ig: immunoglobulin; BHL: bilateral hilar lymphadenopathy; BVB: bronchovascular bundle; VC: vital capacity; \% pred: \% predicted; FEV1: forced expiratory volume in $1 \mathrm{~s}$; FVC: forced VC; PEF: peak expiratory flow; BALF: bronchoalveolar lavage fluid; HPF: high-powered field; F: female; M: male; -: negative; +: positive; DM: diabetes mellitus.

cells were seen in the alveolar interstitium. Furthermore, no granulomata were seen in any of the biopsy specimens. In the bronchial biopsy specimens of all six patients, the IgG4-positive cell count was $>30$ cells per HPF, which is scored as severe according to KAMISAWA et al. [2]. The IgG4-positive/IgG-positive plasma cell ratio was $>30 \%$, which is in agreement with the criteria for IgG4-related lung disease proposed by ZEN et al. [8]. Thus, we diagnosed the lesions in these six patients as central airway involvement of AIP.
Five patients received 4 weeks of $40 \mathrm{mg}$ oral prednisolone, whereas one patient (case 6) did not receive corticosteroid therapy because of the existence of severe cataract and glaucoma. This regimen was followed by a reduction in dose by $5 \mathrm{mg} \cdot \mathrm{week}^{-1}$ over 7 weeks until a daily dose of $5 \mathrm{mg}$ was reached. Blood examinations, chest CTs and PFTs were taken periodically (1-5 months) to follow up on the clinical status of the patients and the stability of the disease. A dramatic clinical improvement was observed in all steroid-treated patients, and 

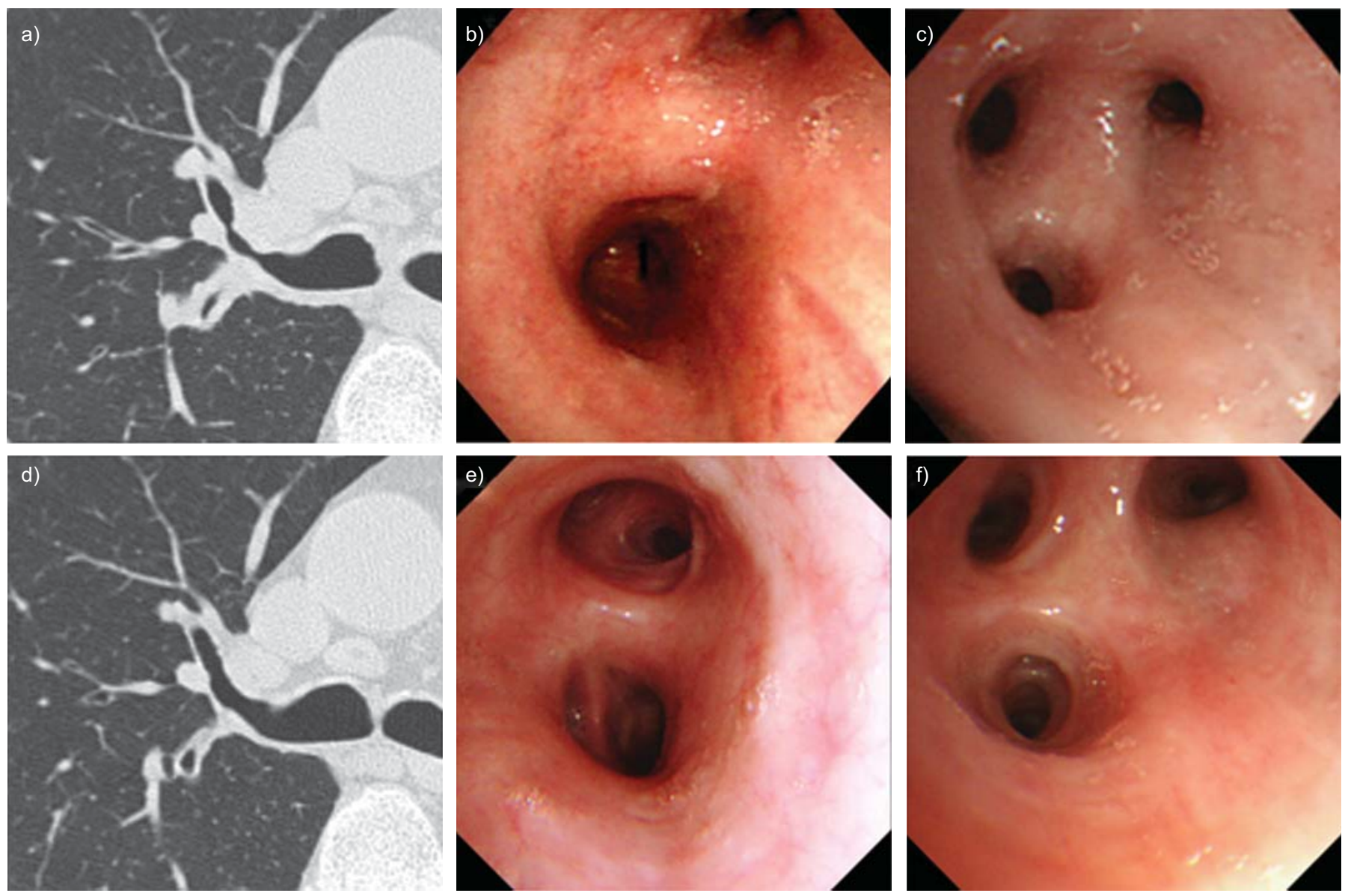

FIGURE 1. Improvements of airway lesions in case 5 after 5 months of corticosteroid treatment. Chest computed tomography (CT) showed d) remarkable improvement of airway narrowing and thickening of the bronchovascular bundles after corticosteroid treatment compared with a) the CT findings before the treatment. Bronchoscopic examination showed remarkable improvement of the irregular stenosis and mucosal oedema in the e) right second spur of the bronchus and f) right upper lobe bronchus after corticosteroid treatment, in comparison with the lesions before the treatment (b and c, respectively).

median (range) serum IgG4 before and after the treatment was significantly reduced from $1,785(773-2,970)$ to 319 (228-969) $\mathrm{U} \cdot \mathrm{mL}^{-1}(\mathrm{p}<0.05)$. PFTs of the five patients showed significant improvements in FEV1, FEV1/FVC and peak expiratory flow after treatment (from 3.08 (2.16-3.60) to 3.57 $(2.75-3.87) \mathrm{L}(\mathrm{p}<0.05), 69.6(60.5-77.6)$ to $78.8(72.0-89.9) \%$ $(\mathrm{p}<0.05)$ and $7.55(3.16-8.09)$ to $9.05(5.34-10.30) \mathrm{L} \cdot \mathrm{s}^{-1}(\mathrm{p}<0.05)$, respectively). Chest $\mathrm{CT}$ imaging revealed a remarkable regression of BHL, and bronchial wall and bronchovascular bundle thickening after corticosteroid treatment (fig. 1d). We performed a bronchoscopic re-examination in one patient (case 5) after treatment and found a remarkable improvement of the irregular stenosis and mucosal oedema in the central airway (fig. $1 \mathrm{e}$ and $\mathrm{f}$ ). Case 6 received low-dose inhaled steroid therapy instead of oral corticosteroid therapy; although the radiological findings and PFTs did not show significant improvements, the patient's cough improved.

It was reported that lung involvement in IgG4-related disease has generally been diagnosed at the time of pancreatic manifestation or afterwards [6]. The one patient (case 6) in this study with a lung imaging abnormality before detection of the pancreatic abnormality is an exception to this rule. Although the radiological and bronchoscopic findings of airway involvement in AIP are characteristic, we think such lesions need to be differentiated from lymphoproliferative disorders and pulmonary sarcoidosis [9]. The TBLB and bronchial biopsy specimens of the involved airways did not show granulomatous lesions. We reported a case of central airway involvement of AIP mimicking sarcoidosis [3] and we considered multicentric Castleman's disease as another differential diagnosis. Although it has been reported that serum ACE concentration is elevated in $40-90 \%$ of patients with clinically active sarcoidosis [9], and serum LDH, CRP and IL-6 levels are elevated in many patients with lymphoproliferative disorders, such as multicentric Castleman's disease [10], it would be difficult to make a final diagnosis by laboratory testing only. Beyond these laboratory tests, clinical, radiological and pathological findings need to be correlated to arrive at the correct diagnosis and distinguish these entities from each other.

To the best of our knowledge, this report is the largest series of patients with biopsy-proven central airway involvement in 
AIP. In conclusion, central airway involvement in AIP is one of the characteristic and steroid-sensitive pulmonary manifestations of AIP.

H. Yamamoto*, M. Yasuo*, M. Ito*, T. Yokoyama*, H. Hamano", T. Uehara", S. Kawakami ${ }^{+}$and K. Kubo* *First Dept of Internal Medicine, "Second Dept of Internal Medicine, "Dept of Laboratory Medicine, and 'Dept of Radiology, Shinshu University School of Medicine, Matsumoto, Japan.

Correspondence: M. Yasuo, First Dept of Internal Medicine, Shinshu University School of Medicine, 3-1-1 Asahi, Matsumoto 390-8621, Japan. E-mail: yasumasa@shinshu-u.ac.jp

Support Statement: This study was funded by an Intractable Disease grant from the Health and Labor Sciences Research Grants of the Ministry of Health, Labor and Welfare (Tokyo, Japan).

Statement of Interest: None declared.

Acknowledgements: We thank K. Tsushima (Shinshu University School of Medicine, Matsumoto, Japan) for finding the peripheral lung involvement with AIP. We also thank Y. Droma (Shinshu University School of Medicine) for preparation of the manuscript. The authors appreciate the valuable advice of H.J. Bogaard (Virginia Commonwealth University, Richmond, VA, USA) for kindly checking the English language usage in our manuscript.

\section{REFERENCES}

1 Kawa S, Hamano H. Clinical features of autoimmune pancreatitis. J Gastroenterol 2007; 42: 9-14.

2 Kamisawa T, Funata N, Hayashi Y, et al. A new clinicopathological entity of IgG4-related autoimmune disease. J Gastroenterol 2003; 38: 982-984.

3 Ito M, Yasuo M, Yamamoto $H$, et al. Central airway stenosis in a patient with autoimmune pancreatitis. Eur Respir J 2009; 33: 680-683.

4 Fujinaga Y, Kadoya M, Kawa S, et al. Characteristic findings in images of extra-pancreatic lesions associated with autoimmune pancreatitis. Eur J Radiol 2010; 76: 228-238.

5 Tsushima K, Tanabe T, Yamamoto $\mathrm{H}$, et al. Pulmonary involvement of autoimmune pancreatitis. Eur J Clin Invest 2009; 39: 714-722.

6 Shrestha B, Sekiguchi H, Colby TV, et al. Distinctive pulmonary histopathology with increased IgG4-positive plasma cells in patients with autoimmune pancreatitis: report of 6 and 12 cases with similar histopathology. Am J Surg Pathol 2009; 33: 1450-1462.

7 Siddiqui S, Martin JG. Structural aspects of airway remodeling in asthma. Curr Allergy Asthma Rep 2008; 8: 540-547.

8 Zen Y, Inoue D, Kitao A, et al. IgG4-related lung and pleural disease: a clinicopathologic study of 21 cases. Am J Surg Pathol 2009; 33: 1886-1893.

9 Costabel U. Sarcoidosis: clinical update. Eur Respir J 2001; 18: Suppl. 32, 56s-68s.

10 Sato Y, Kojima M, Takata K, et al. Systemic IgG4-related lymphadenopathy: a clinical and pathologic comparison to multicentric Castleman's disease. Mod Pathol 2009; 22: 589-599.

DOI: $10.1183 / 09031936.00017611$ 